NBI-HE-97-34

hep-th/9709047

\title{
Deformation of Super Virasoro Algebra in Noncommutative Quantum Superspace
}

\author{
Haru-Tada Sato \\ The Niels Bohr Institute, University of Copenhagen \\ Blegdamsvej 17, DK-2100 Copenhagen, Denmark
}

\begin{abstract}
We present a twisted commutator deformation for $N=1,2$ super Virasoro algebras based on $G L_{q}(1,1)$ covariant noncommutative superspace.
\end{abstract}

PACS: 02.10.Jf, 11.17.+y

Keywords: Virasoro algebra, algebra deformation, quantum group, noncommutative space

${ }^{1}$ sato@nbi.dk 
Several years ago, a deformation of Virasoro algebra was studied based on a deformed Heisenberg oscillator or a deformed differential operator. The deformation comprises a particular type of twisted commutators, firstly proposed by Curtright and Zachos [1], and it so far has nothing to do with a Hopf algebra structure, which is an important notion for quantum deformation. In this sense, this deformation differs from ordinary quantum deformation, however is an algebra of well-defined differential operators; i.e., oscillators. We refer to this algebra as $C Z$ algebra in this paper, and a review can be found in [2] (where a connection to other deformation [3] is discussed as well).

The physical importance of $\mathrm{CZ}$ algebra seems to lie in the following regime. The idea of deformed Heisenberg algebra may be useful for constructing various objects in an algebraic manner, and would be fundamental to discuss a quantum excitation of deformed fluctuating vacuum. Thus a deformed symmetry algebra of Virasoro type should naturally be expressed in terms of a deformed oscillator algebra. For example, it is found that a $W$-algebra extension [4] of $\mathrm{CZ}$ algebra emerges from a finite (deformed oscillator) representation of $c=1$ matrix model [5]. This is the example of physical implication of CZ algebra in a fluctuating system.

The generators of $\mathrm{CZ}$ algebra can also be constructed on a quantum group basis (8) covariant under a matrix action, where matrices form a (quantum) group and their matrix entries are noncommutative. Accordingly, any component of the basis can generally be a noncommutative quantity and become suitable to formulate such a fluctuating object where the notion of commutable point may not be allowed. We shall call this noncommutative space "quantum space".

Our intension is to generalize this approach into a supersymmetric case introducing a basis covariant under a super quantum group action. The purpose of this letter is to present a simple supersymmetric extension of the CZ algebra, allowing $U(1)$ element's participation. This is a concise deformation compared to the previous awkward results [6], and is nontrivial since there is no well-established method to construct a super CZ algebra.

In [6], we discussed a method how to extend the deformed Virasoro (CZ) algebra into 
a superalgebra. The bosonic elements of the deformed algebra satisfy

$$
\begin{aligned}
& {\left[F_{n}, F_{m}\right]_{(m-n)}=0,} \\
& {\left[L_{n}^{C Z}, F_{m}\right]_{(m-n)}=-q^{-n}[m] F_{n+m},} \\
& {\left[L_{n}^{C Z}, L_{m}^{C Z}\right]_{(m-n)}=[n-m] L_{n+m}^{C Z},}
\end{aligned}
$$

where $[x]=\left(q^{x}-q^{-x}\right) /\left(q-q^{-1}\right)$ and twisted (anti-)commutators are defined as

$$
[A, B\}_{(a)}=q^{a} A B \pm q^{-a} B A
$$

The $F_{n}$ is an element of a deformed $U(1)$ algebra [7], and $L_{n}^{C Z}$ represents the deformed Virasoro algebra (CZ algebra). These operators can be defined in terms of bosonic quantum space operators [8]. For the purpose of supersymmetric extension, these operators should be defined on a (noncommutative) quantum superspace, and we make use of the following $G L_{q}(1,1)$ covariant quantum superspace [9]

$$
\begin{aligned}
& (\theta)^{2}=\left(\partial_{\theta}\right)^{2}=0, \quad x \theta=q \theta x, \quad \partial_{x} \partial_{\theta}=q^{-1} \partial_{\theta} \partial_{x} \\
& \partial_{x} x=1+q^{-2} x \partial_{x}, \quad \partial_{\theta} \theta=1-\theta \partial_{\theta}+\left(q^{-2}-1\right) x \partial_{x} \\
& \partial_{x} \theta=q^{-1} \theta \partial_{x}, \quad \partial_{\theta} x=q^{-1} x \partial_{\theta}
\end{aligned}
$$

where $x$ and $\theta$ are the bosonic and fermionic (noncommutative) coordinates. In the previous paper, we introduced the operator $\left(\mu=\partial_{x} x-x \partial_{x}\right)$ which commutes with $\theta$ and $\partial_{\theta}$ as a scaling operator, however this time, we consider the scaling operator such that (for a real number $a$ )

$$
\begin{aligned}
& \lambda x=q^{-2} x \lambda, \quad \lambda \partial_{x}=q^{2} \partial_{x} \lambda, \\
& \lambda \theta=q^{2 a} \theta \lambda \quad \lambda \partial_{\theta}=q^{-2 a} \partial_{\theta} \lambda .
\end{aligned}
$$

The $\lambda$ operator is realized by

$$
\lambda=\mu+\left(q^{2 a}-1\right) \theta \partial_{\theta} .
$$

According to the program [6] to construct a super CZ algebra, we first define the $G_{r}$ operator

$$
G_{r}=\lambda^{-1 / 2} x^{r+\frac{1}{2}}\left(\partial_{\theta}-\theta \partial_{x}\right)
$$


Here we fix $a=-1 / 2$ imposing the relation

$$
\lambda G_{r}=q^{-2 r} G_{r} \lambda
$$

and we thus reckon

$$
G_{r} G_{s}=-q^{r+s+3 / 2} x^{r+s+1} \partial_{x}-q^{r+2}\left[s+\frac{1}{2}\right] x^{r+s} \theta \partial_{\theta} .
$$

Remarkably, the following twisted anti-commutator can be cast into the form which depends only on $r+s$ (up to an overall factor)

$$
\left\{G_{r}, G_{s}\right\}_{\left(\frac{s-r}{2}\right)}=-q^{5 / 2+r+s}\left(q^{\frac{s-r}{2}}+q^{\frac{r-s}{2}}\right)\left(q^{-1} x^{r+s+1} \partial_{x}+\frac{1-q^{-r-s-1}}{q-q^{-1}} x^{r+s} \theta \partial_{\theta}\right) .
$$

One may express RHS of Eq.(12) by two bases, for example $L_{n}^{C Z}$ and $F_{n}$, as discussed in [6], however we here simply propose the bosonic operator $L_{n}$ defined in terms of the RHS; namely

$$
L_{n}=-q^{-1} x^{n+1} \partial_{x}-A_{n} x^{n} \theta \partial_{\theta}, \quad A_{n}=\frac{1-q^{-n-1}}{q-q^{-1}} .
$$

This $L_{n}$ is different from the $L_{n}^{C Z}$ operator

$$
L_{n}^{C Z}=-q^{-1} x^{n+1} \partial_{x}-A_{n}^{C Z} x^{n} \theta \partial_{\theta}, \quad A_{n}^{C Z}=c_{1} q^{-2 n}+c_{2}
$$

where $c_{i}(i=1,2)$ are arbitrary constants, and the relation between these two Virasorolike operators is

$$
L_{n}^{C Z}=L_{n}+\epsilon_{n} F_{n}, \quad \epsilon_{n}=A_{n}-A_{n}^{C Z},
$$

where the deformed $U(1)$ operator appears

$$
F_{n}=x^{n} \theta \partial_{\theta}
$$

We therefore find the algebras among $L_{n}$ and $F_{n}$ besides the superalgebra of $G_{r}$;

$$
\begin{gathered}
\left\{G_{r}, G_{s}\right\}_{\left(\frac{s-r}{2}\right)}=q^{5 / 2+r+s}\left(q^{\frac{s-r}{2}}+q^{\frac{r-s}{2}}\right) L_{r+s}, \\
{\left[L_{n}, G_{r}\right]_{\left(r-\frac{n}{2}\right)}=q^{-n}\left[\frac{n}{2}-r\right] G_{n+r},}
\end{gathered}
$$




$$
\begin{aligned}
& {\left[L_{n}, F_{m}\right]_{(m-n)}=-q^{-n}[m] F_{n+m},} \\
& {\left[L_{n}, L_{m}\right]_{(m-n)}=[n-m] L_{n+m}+a_{n m} F_{n+m},}
\end{aligned}
$$

where

$$
a_{n m}=q^{-n-m-1}\left(q-q^{-1}\right)\left[\frac{n}{2}\right]\left[\frac{m}{2}\right]\left[\frac{n-m}{2}\right] .
$$

As expected, $L_{n}$ is not an independent operator since we have the relation from Eq.(11)

$$
L_{n}=q^{-5 / 2-n} G_{n / 2}^{2} .
$$

Note that $\lambda$ is related to $L_{0}$ by

$$
\lambda=1+\left(q-q^{-1}\right) L_{0},
$$

and also that $\lambda$ plays the role of a central element

$$
\left[\lambda, E_{n}\right]_{(n)}=0
$$

where $E_{n}$ stands for an arbitrary element of $G_{n}, L_{n}$ or $F_{n}$. This deformed superalgebra contains the following $q$-su $(1,1)$ and $q$-osp $(1,2)$ algebras of the CZ-type deformation: For the $s u(1,1)$ part, (noticing $a_{n m}=0$ for $n, m=0, \pm 1$ ), we have

$$
\left[L_{n}, L_{m}\right]_{(m-n)}=[n-m] L_{n+m}, \quad(n, m=0, \pm 1)
$$

and for the $\operatorname{osp}(1,2)$ part $(r= \pm 1 / 2)$, we have

$$
\begin{aligned}
& \left\{G_{r}, G_{-r}\right\}_{(-r)}=q^{5 / 2}\left(q^{r}+q^{-r}\right) L_{0}, \quad\left(G_{ \pm 1 / 2}\right)^{2}=q^{5 / 2 \pm 1} L_{ \pm 1} \\
& {\left[L_{0}, G_{r}\right]_{(r)}=-[r] G_{r}}
\end{aligned}
$$

Several remarks are now in order. First one is on the Jacobi identity constraints [10;

$$
\begin{aligned}
& {\left[G_{t},\left\{G_{r}, G_{s}\right\}_{\left(\frac{s-r}{2}\right)}\right]_{\left(\frac{r+s}{2}-t\right)}+\text { c.p. }=0,} \\
& {\left[F_{n},\left[F_{m}, F_{l}\right]_{(l-m)}\right]_{(m+l-2 n)}+\text { c.p. }=0,} \\
& {\left[L_{n},\left[F_{m}, F_{l}\right]_{(l-m)}\right]_{(m+l-2 n)}+\text { c.p. }=0,}
\end{aligned}
$$




$$
\begin{aligned}
& {\left[L_{t},\left\{G_{r}, G_{s}\right\}_{\left(\frac{s-r}{2}\right)}\right]_{(r+s-t)}-\left\{G_{s},\left[L_{t}, G_{r}\right]_{\left(r-\frac{t}{2}\right)}\right\}_{\left(\frac{r+t-3 s}{2}\right)}} \\
& +\left\{G_{r},\left[G_{s}, L_{t}\right]_{\left(\frac{t}{2}-s\right)}\right\}_{\left(\frac{s+t-3 r}{2}\right)}=0
\end{aligned}
$$

and so forth, where c.p. means the cyclic permutations as usual. The first two relations $(28),(29)$ are satisfied by Eqs.(17),(18) and (1). On the other hand, the latter two relations give rise to the following constraint equations, which are similar to the (bosonic) $\mathrm{CZ}$ algebra case [1]

$$
\begin{gathered}
{[m]\left[F_{l}, F_{n+m}\right]_{(n+m)}=[l]\left[F_{m}, F_{l+n}\right]_{(l+n)}} \\
q^{5 / 2+r+s}\left(q^{\frac{r-s}{2}}+q^{\frac{s-r}{2}}\right)\left[L_{t}, L_{r+s}\right]_{\left(\frac{r+s}{2}-t\right)}-q^{-t}\left[\frac{t}{2}-r\right]\left\{G_{s}, G_{t+r}\right\}_{\left(\frac{r+t-3 s}{2}\right)} \\
-q^{-t}\left[\frac{t}{2}-s\right]\left\{G_{r}, G_{t+s}\right\}_{\left(\frac{s+t-3 r}{2}\right)}=0 .
\end{gathered}
$$

The $q$-su $(1,1)$ and $q$-osp $(1,2)$ parts do not give rise to any constraint. These constraints should be satisfied when an explicit realization is applied, and ensure the associativity of operator algebra. For example, those are of course satisfied when the differential operators shown above are substituted. According to this feature, we may transform the constraints into algebra forms. The constraint algebras, with Eq.(22), are thus

$$
\begin{gathered}
F_{n} F_{m}=q^{3 n+m} \lambda F_{n+m}, \\
\left\{G_{r}, G_{s}\right\}_{\left(\frac{3 s-r}{2}\right)}=q^{5 / 2+r+s}\left(q^{\frac{3 s-r}{2}}+q^{\frac{r-3 s}{2}}\right) L_{r+s}+q^{3 / 2}\left(q-q^{-1}\right)[r]\left[\frac{s-r}{2}\right] F_{r+s} .
\end{gathered}
$$

We show two examples of the usage of these constraint algebras; (i) One should note that Eq.(20) can be checked by using Eqs.(22), (17),(18) and (35) in order

$$
\begin{aligned}
& {\left[L_{n}, L_{m}\right]_{(m-n)}=q^{-(n+m+5)}\left(q^{3(m-n) / 4} G_{n / 2}\left\{G_{n / 2}, G_{m / 2}\right\}_{\left(\frac{m-n}{4}\right)} G_{m / 2}\right.} \\
& \quad-q^{(m-n) / 4} G_{n / 2} G_{m / 2}\left\{G_{n / 2}, G_{m / 2}\right\}_{\left(\frac{m-n}{4}\right)}+\left\{G_{n / 2}, G_{m / 2}\right\}_{\left(\frac{m-n}{4}\right)} q^{(n-m) / 4} G_{m / 2} G_{n / 2} \\
& \left.\quad-q^{3(n-m) / 4} G_{m / 2}\left\{G_{n / 2}, G_{m / 2}\right\}_{\left(\frac{m-n}{4}\right)} G_{n / 2}\right) \\
& \quad=q^{-(n+m+5) / 2}\left(q^{(n-m) / 4}+q^{(m-n) / 4}\right) \\
& \quad \times\left(q^{(m-n) / 2} G_{n / 2}\left[L_{\frac{n+m}{2}}, G_{m / 2}\right]_{\left(\frac{m-n}{4}\right)}+q^{(n-m) / 2}\left[L_{\frac{n+m}{2}}, G_{m / 2}\right]_{\left(\frac{m-n}{4}\right)} G_{n / 2}\right) \\
& \quad=q^{-n-m-5 / 2}\left(q^{(n-m) / 4}+q^{(m-n) / 4}\right)\left[\frac{n-m}{4}\right]\left\{G_{n / 2}, G_{m+n / 2}\right\}_{\left(\frac{m-n}{2}\right)} .
\end{aligned}
$$


(ii) Assuming these constraints (in other words, no central extension for constraint algebras), we may find central extensions utilizing the Jacobi identities (28) and (29)

$$
\begin{gathered}
\left\{G_{r}, G_{s}\right\}_{\left(\frac{s-r}{2}\right)}=q^{5 / 2+r+s}\left(q^{\frac{s-r}{2}}+q^{\frac{r-s}{2}}\right) L_{r+s}+f_{1}(r) \lambda \delta_{r+s, 0} \\
{\left[F_{n}, F_{m}\right]_{(m-n)}=f_{2}(n) \lambda^{2} \delta_{n+m, 0}}
\end{gathered}
$$

where we leave the coefficients $f_{i}$ undetermined. These forms of central terms are consistent with Eqs.(31) and (30) as well.

Secondly, let us decompose the above set of algebras into a $N=2$ algebra. The decomposition may be performed by

$$
G_{r}=G_{r}^{-}+G_{r}^{+}
$$

with the two operators

$$
G_{r}^{-}=\lambda^{-1 / 2} x^{r+1 / 2} \partial_{\theta}, \quad G_{r}^{+}=-\lambda^{-1 / 2} x^{r+1 / 2} \theta \partial_{x}
$$

The centerless $N=2 \mathrm{CZ}$ algebra is therefore

$$
\begin{gathered}
\left\{G_{r}^{+}, G_{s}^{-}\right\}=q^{5 / 2+r+s} L_{r+s}+q^{3 / 2+\frac{r-s}{2}}\left[\frac{r-s}{2}\right] F_{r-s}, \\
\left\{G_{r}^{+}, G_{s}^{+}\right\}=\left\{G_{r}^{-}, G_{s}^{-}\right\}=0 \\
{\left[L_{n}, G_{r}^{ \pm}\right]_{\left(r-\frac{n}{2}\right)}=q^{-n}\left[\frac{n}{2}-r\right] G_{n+r}^{ \pm},} \\
{\left[F_{n}, G_{r}^{+}\right]_{(a, b)}=q^{n+2+a} \lambda G_{n+r}^{+}, \quad\left[F_{n}, G_{r}^{-}\right]_{(a, b)}=-q^{n+2 r+1+b} \lambda G_{n+r}^{-} .}
\end{gathered}
$$

where $a$ and $b$ are ambiguous constants, and

$$
[A, B]_{(a, b)}=q^{a} A B-q^{b} B A
$$

To close this discussion, we finally put a brief comment on a coproduct structure. Originally, all these algebras are not defined in an abstract sense, but obtained as differential operator algebras acting on functions of quantum space variables. In the similar sense, we can derive some coproducts as Leipnitz rules for those differential operators, 
acting on a quantum superspace tensor bases $V_{m n}^{1} \otimes V_{m n}^{2}$ where $V_{m n}^{1}=x^{m} \theta^{n}$ etc. For example, the operation rules (in other words, Leipnitz rules)

$$
\begin{aligned}
& \partial_{x} x^{n}=q^{-2 n} x^{n} \partial_{x}+q^{-n+1}[n] x^{n-1}, \\
& \lambda x^{n}=q^{-2 n} x^{n} \lambda,
\end{aligned}
$$

can be re-cast in the following forms of coproduct

$$
\begin{aligned}
\Delta\left(\partial_{x}\right) & =\partial_{x} \otimes 1+\lambda \otimes \partial_{x} \\
\Delta(\lambda) & =\lambda \otimes \lambda .
\end{aligned}
$$

Similarly, we have

$$
\begin{aligned}
& \Delta\left(L_{n}\right)=L_{n} \otimes 1+\lambda \otimes L_{n}, \\
& \Delta\left(F_{n}\right)=F_{n} \otimes 1+\lambda \otimes F_{n},
\end{aligned}
$$

and so forth. It is not so clear whether or not a fermionic operator (such as $\partial_{\theta}$ and $G_{r}$ ) can be organized into a unique coproduct expression. The relation (22) would seem to suggest the structure

$$
\Delta\left(G_{r}\right)=G_{r} \otimes 1+\lambda^{1 / 2} \otimes G_{r}
$$

A check whether this works or not might be the following. Notice that Eqs.(50) and (52) for $n=0, r= \pm \frac{1}{2}$ are the same coproduct structure as the one of the following Woronowitz type $q$-osp $(1,2)$

$$
\begin{aligned}
& \tilde{G}_{-1 / 2} \tilde{G}_{1 / 2}+q^{-1 / 2} \tilde{G}_{1 / 2} \tilde{G}_{-1 / 2}=\alpha(q) \tilde{L}_{0} \\
& \tilde{L}_{0} \tilde{G}_{-1 / 2}-q \tilde{G}_{-1 / 2} \tilde{L}_{0}=q \gamma(q) \tilde{G}_{-1 / 2} \\
& \tilde{L}_{0} \tilde{G}_{1 / 2}-q^{-1} \tilde{G}_{1 / 2} \tilde{L}_{0}=-\gamma(q) \tilde{G}_{1 / 2} .
\end{aligned}
$$

The question then becomes whether or not a map between this algebra and ours keeps the coproduct structure unchanged. If one finds a mapping relation of our algebra into the standard $q$-osp $(1,2)$ algebra [12]

$$
\left[H, V_{ \pm}\right]= \pm \frac{1}{2} V_{ \pm}, \quad\left\{V_{+}, V_{-}\right\}=-\frac{1}{4} \frac{p^{2 H}-p^{-2 H}}{p-p^{-1}}
$$


our algebra will be transformed into the algebra (53) through the map

$$
\begin{aligned}
& \tilde{L}_{0}=q \gamma \frac{q^{2 H}-1}{q-1}, \\
& \tilde{G}_{-1 / 2}=2 \sqrt{q \alpha \gamma} V_{+} q^{H / 2}, \quad \tilde{G}_{1 / 2}=2 \sqrt{q \alpha \gamma} q^{H / 2} V_{-}, \\
& p=q^{1 / 2} .
\end{aligned}
$$

It is known that the coproduct structure for (54) can be mapped into the one for (53), and we expect that the problem would be solved in the similar way.

\section{Acknowledgement}

The author would like to thank N. Aizawa and T. Kobayashi for discussions.

\section{References}

[1] T. Curtright and C. Zachos, Phys. Lett. B243 (1990) 237.

[2] M. Chaichian and P. Presnajder, Nucl. Phys. B482 (1996) 466 (hep-th/9603064).

[3] M. Chaichian and P.P. Presnajder, Phys. Lett. B277 (1992) 109;

H. Sato, Nucl. Phys. B393 (1993) 442;

A.A. Belov and K.D. Chaltikian, Mod. Phys. Lett. A8 (1993) 1233;

H-T. Sato, Nucl. Phys. B471 (1996) 553;

E. Batista, J.F. Gomes and I.J. Lautenschleguer, J. Phys. A29: Math. Gen. (1996) 6281 (q-alg/9603004);

R. Kemmoku and S. Saito, Phys. Lett. B319 (1993) 471; hep-th/9411027.

[4] C.-Z. Zha, J. Math. Phys. 35 (1994) 517.

[5] A. Jevicki and A. van Tonder, Mod. Phys. Lett. A11 (1996) 1397.

[6] N. Aizawa, T. Kobayashi and H.T. Sato, to appear in Int. Jour. Mod. Phys. A (hep-th/9706176). 
[7] idem. Mod. Phys. Lett. A11 (1996) 37.

[8] A.El Hassouni, Y. Hassouni, E.H. Tahri and M. Zakkari, Mod. Phys. Lett. A10 (1995) 2169.

[9] T. Kobayashi, Z. Phys. C60 (1993) 101.

[10] M. Chaichian, P. Kulish and J. Lukierski, Phys. Lett. B237 (1990) 401.

[11] H. Sato, Prog. Theor. Phys. 89 (1993) 531;

N. Aizawa and H. Sato, Phys. Lett. B256 (1991) 185.

[12] P.P Kulish and N.Yu. Reshetikhin, Lett. Math. Phys. 18 (1989) 143;

R. Floreanini and L. Vinet, J. Phys. A23: Math. Gen. (1990) L1019;

H. Saleur, Nucl. Phys. B336 (1990) 363. 